\title{
Compradores compulsivos en la Ciudad de México
}

\author{
Dra. Laura Estela Fischer de la Vega
}

Facultad de Contaduría y Administración UNAM

Mtro. Jorge Ángel Espejo Callado

Universidad Autónoma del Estado de Morelos

\author{
Dra. Adriana Méndez Wong \\ Dr. Jesús Francisco Mellado Siller
}

Universidad Autónoma de Coahuila

doi: 10.19044/esj.2016.v12n2p21 URL:http://dx.doi.org/10.19044/esj.2016.v12n2p21

\begin{abstract}
Buying behavior constitutes an essential activity of individuals in contemporary society. Buying behavior occurs in response to a complex phenomenon normally preceded by what has been termed "previous activity" and does not end with satisfying some need. Once purchasing occurs the individual experiences a series of feelings and thoughts of satisfaction or frustration related to the purchased object or service (Fischer \& Espejo, 2011). The objective of the present study was to determine compulsive buying levels from the conceptual standpoint proposed by Valence, D’Astous \& Fortier which incude three factors: tendency to spend, buying sense of urgency, and feelings of guilt (Valence, 1988). The present study was the third of a series and used qualitative-explanatory methodology. A total of 1,385 adult residents of Mexico City's metropolitan area: 60.1\% females and $39.9 \%$ males participated. Results showed that most participants engaged in mild compulsive buying.
\end{abstract}

Keywords: Compulsive buying, impulsiveness, compulsiveness scale

\section{Resumen}

En nuestros días, el comportamiento de compra es parte de la esencia de la vida del individuo. La compra es una respuesta a un fenómeno muy complejo que inicia en una etapa llamada "actividad previa" y que no culmina con la satisfacción de la necesidad, ya que una vez realizada se produce una instancia post-compra en la que nacen sentimientos y pensamientos de satisfacción o frustración con relación al producto o servicio adquirido (Fischer \& Espejo 2011). El presente estudio tuvo como objetivo determinar los niveles de compulsividad en la compra desde la 
perspectiva conceptual de Valence, d'Astous \& Fortier en sus factores: Tendencia a gastar, Urgencia de compra y Sentimientos de culpabilidad (Valence, 1988). El estudio incluyó a 1,385 participantes adultos residentes en el área metropolitana de la Ciudad de México, 60.1\% mujeres y 39.9\% hombres, encontrando resultados que muestran que en general la población tiene características de comprador compulsivo, aunque ligeras.

Palabras claves: Compra Compulsiva, Compra por Impulso, Escala de Compulsividad

\section{Introducción}

Desde hace varias décadas las empresas se preocupan por conocer el comportamiento de los consumidores con el propósito de acercarles sus productos o servicios de la forma más idónea. La conducta del consumidor es muy compleja, autores como Kotler \& Armstrong (Kotler 2007), Stanton, Etzel \& Walker (Stanton, 2007), Arellano (Arellano, 2002), Blackwell , Miniard \& Engel (Blackwell, 2002), Fischer \& Espejo (Fischer, 2011), Garnica \& Maubert (Garnica, 2009) y Hawkins \& Best \& Coney (Hawkins, 2004), entre muchos otros, han estudiado al consumidor desde el punto de vista de la mercadotecnia, encontrando una gama de aspectos culturales, sociales, de personalidad y del medio ambiente, que influyen de diferente forma en el momento de decidir la compra. Entre los consumidores existen aquellos que consumen de forma responsable, pero existen también quienes compran en forma tan impulsiva que su conducta llega a convertirse en compulsiva y termina siendo adictiva.

\section{Marco teórico}

Dentro del esquema de las tendencias de autorregulación ${ }^{1}$, el comportamiento de compra se clasifica como impulsivo, compulsivo y adictivo (Bandura, 1986). El nivel más alto de autorregulación va de la mano con el nivel más bajo de susceptibilidad mostrado por los consumidores hacia la compra: se trata del nivel de impulsividad, que es el menos dañino. En el otro extremo, el nivel más bajo de autorregulación conduce al nivel más alto de vulnerabilidad de los consumidores hacia la compra, se clasifica como adicción y es el nivel más dañino (LaRose, 2002). Por su parte, la Asociación Americana de Psiquiatría (AAP, 1985) define la compra compulsiva como "una conducta aparentemente intencional y repetitiva que se realiza de acuerdo a reglas o de forma estereotipada", se trata de un comportamiento obsesivo-compulsivo que Goldenson (Goldenson, 1984)

\footnotetext{
${ }^{1}$ La autorregulación es un mecanismo del ser humano que le permite mantenerse en balance psicológico.
} 
describe como un trastorno de ansiedad y angustia, que interfiere con la capacidad del individuo para funcionar. Workman \& Paper describen a las obsesiones como ideas e impulsos recurrentes, persistentes, sin sentido y repugnantes, que no pueden ser ignorados o suprimidos por la persona. Para los autores, las compulsiones son generalmente comportamientos excesivos y rituales orientados a aliviar la tensión, la ansiedad, o el malestar suscitado por un pensamiento u obsesión entrometida (Workman, 2010).

La compra compulsiva no se considera sinónimo de adquirir bienes en cantidades excesivas, aunque comprar grandes cantidades de productos que no se necesitan y que con frecuencia no se pueden pagar, es una característica de la adicción a la compra (García, 2007). Desde la perspectiva de O'Guinn \& Faber la compra compulsiva es un comportamiento de compra crónica, repetitiva, que se produce como respuesta a eventos o sentimientos negativos, como el aburrimiento, el estrés, la tensión o la ansiedad (O'Guinn, 1987). Valence, d'Astous \& Fortier, explican que la ansiedad en el consumidor se manifiesta a través de un impulso acompañado de excitación emocional, que provoca espontaneidad y lo anima a reducir la tensión mediante la compra compulsiva (Valence, 1988), por consiguiente, se argumenta que la principal motivación de un comprador para mostrar un comportamiento compulsivo de compra es escapar de la ansiedad (Roberts, 2004), aliviar el estrés y, finalmente, sentirse feliz. También se ha sugerido que la ansiedad experimentada por el individuo puede ser a la vez causa y efecto de la compra compulsiva (Roberts J. J., 2001). Valence, d'Astous \& Fortier sugieren dos grandes grupos de factores que pueden desencadenar en el individuo la compra compulsiva por ansiedad: (a) los factores sociales: cultura, entorno comercial, comunicación publicitaria, etc., que incentivan los ideales materialistas, y (b) los factores individuales: una fuerte activación emocional (aumento de la tensión psicológica), un control cognitivo alto (reconocimiento de que la compra reducirá la tensión) y una alta reactividad (búsqueda para reducir la tensión) (Valence, 1988). Luna-Arocas señala que esto explica por qué la diferencia entre la compra compulsiva y la compra impulsiva procede del proceso cognitivo: la persona tiende a asociar un rápido desajuste de su estado afectivo con el acto de compra (Luca-Arocas, 2000).

En los últimos años, los investigadores que más interés han mostrado por entender el comportamiento de compra compulsiva han construido, desde diferentes perspectivas, escalas para diagnosticar y analizar la conducta compulsiva de compra. La primera escala propuesta para estudiar los constructos subyacentes del comportamiento compulsivo de compra fue desarrollada por Valence, d'Astous y Fortier (Valence, 1988), tiene sus bases en la perspectiva conceptual propuesta por Faber, O'Guinn y Krych (Faber, 1988). En su primera versión, la escala considera cuatro dimensiones; la 
primera es identificada como "tendencia a gastar", e implica que un comprador compulsivo debe exhibir una mayor propensión a gastar que un comprador no compulsivo; la segunda dimensión se ocupa de la respuesta a fuertes deseos de compra, un individuo que exhibe un comportamiento compulsivo de compra siente que las motivaciones o urgencias de compra son irresistibles o están más allá de su control, mientras que los compradores no compulsivos no ven tales motivaciones o urgencias como incontrolables; la tercera dimensión asociada a la compra compulsiva es el sentimiento de culpabilidad posterior a la compra, diversos estudios han reportado evidencia de que las personas que compran en forma compulsiva a menudo sienten remordimientos por sus conductas (Faber, 1988); por último, la cuarta dimensión se refiere al entorno familiar, sin embargo, en la versión final de la escala, esta dimensión se eliminó por su débil consistencia interna. La escala de Valence y colegas ha sido aplicada en diversos estudios cuyos resultados han registrado un alfa de Cronbach entre .78 y .92, estos niveles sugieren que la escala asocia fuertemente los motivadores relacionados con la compra compulsiva, es por ello que para diversos investigadores existe evidencia de que la escala se inclina más hacia la identificación del "shopping" compulsivo y no a las tendencias del "buying” compulsivo, dado que los sujetos impulsivos difieren significativamente de los compulsivos en la implicación con la compra y en la motivación de compra pero no en el materialismo ni en la utilización de las tarjetas de crédito.

En 1992 Faber \& O'Guinn, desde una perspectiva fenomenológica, desarrollaron una escala para identificar a los compradores compulsivos dentro de una población general, la escala contiene los constructos autoestima, materialismo, y uso de tarjetas de crédito por estar asociados con la compra compulsiva; la escala se compone de siete ítems, las variables incluidas son personalidad, motivaciones para la compra, y consecuencias de la compra compulsiva. La escala correlaciona las actitudes hacia el dinero, pero no lo hace con el materialismo; la escala de Faber mide el comportamiento de compra compulsiva, mientras que la escala de Valence mide tendencias compulsivas de compra (Faber 1992).

En 1993, Edwards identificó cinco niveles distintos de compra compulsiva, en la que todos los consumidores podríamos ser clasificados, estos niveles son, de menor a mayor nivel de gravedad: (a) No compulsivos, se trata de las personas que realizan exclusivamente las compras planeadas cuando se necesitan los productos/servicios; (b) Recreacional, personas que de vez en cuando hacen compras impulsivas no planificados, a menudo en un esfuerzo por mejorar el estado de ánimo; (c) Frontera, al igual que los compradores recreacionales, estas personas de vez en cuando compran artículos para mejorar el estado de ánimo, pero pueden sentirse culpables después de la compra de artículos que no se necesitan; (d) Compulsivo, 
personas que con frecuencia compran artículos para aliviar el estrés o la ansiedad, a menudo se sienten culpables después de la compra de productos/servicios que no se necesitan, y sufren emociones negativas y consecuencias financieras por la conducta de compra; (e) Adictos, personas que experimentan un poderoso y continuo impulso para comprar productos/servicios, y es tan fuerte el impulso que a menudo descuidan otras obligaciones, posponen o cancelan otras actividades para actuar en este impulso (Edwards, 1992).

En 1996, Monahan (Monahan 1996) y colegas modificaron la escala Yale-Brown (Goodman, 1989) para crear la versión YBOCS-Compra, con el propósito de evaluar las cogniciones y comportamientos asociados con la compra compulsiva. El instrumento consta de 10 ítems, de los cuales 5 califican preocupaciones y 5 comportamientos; los ítems se valoran en una escala que corre de 0 a 4 y las puntuaciones pueden variar entre 0 y 40.

En 2008, Ridgway propone su escala para medir el comportamiento de compra compulsiva, la diferencia con sus predecesores es variada. Ridgway aprecia a la compra compulsiva como un trastorno de ansiedad obsesivo-compulsivo, pero también la ve como un trastorno del control de los impulsos, además, las escalas anteriores casi siempre incluyen cuestiones financieras y de deuda. Gracias a Ridgway hoy se sabe que existen compradores compulsivos sin problemas financieros.

\section{Metodología \\ Objetivos}

Se realizó un estudio cuantitativo-explicativo transversal, con el objetivo de determinar niveles de compra compulsiva en los habitantes de la Ciudad de México, desde la perspectiva conceptual de Valence, d'Astous \& Fortier en sus factores: Tendencia a gastar, Urgencia de compra y Sentimientos de culpabilidad (Valence, 1988); y considerando la estructura de la escala de medición propuesta por Edwards (Edwards, 1992).

Un segundo objetivo ha sido comparar empíricamente las medidas de compra compulsiva que proporcionan las escalas de la Valence, d'Astous \& Fortier (Valence, 1988), Faber y O'Guinn (1992) y Ridgway (2008) con el propósito de evaluar su dimensionalidad y rendimiento relativo en la identificación de los compradores compulsivos.

\section{Diseño de la investigación}

Muestra. La investigación, utilizó una muestra de 1,385 participantes residentes en el área metropolitana de la Ciudad de México, 60.1\% mujeres y 39.9\% hombres; tomando la población de cada estrato se determinó el número de encuestas a realizar por rango de edades, como se muestra en la tabla 1 (Babbie, 1988), (Scheaffer, 2007). 
Tabla 1. Estratificación de la muestra (elaboración propia)

Rango de edad
10 a 14 años
15 A 19
20 A 24
25 A 29
30 A 34
35 A 39
40 A 44
45 A 49
50 A 54
55 A 59
$60+$

Total

$$
\begin{gathered}
\text { Población }^{2} \\
660,345 \\
723,372 \\
753,404 \\
726,698 \\
702,642 \\
722,214 \\
629,563 \\
555,481 \\
506,310 \\
392,186 \\
1,201,674 \\
\mathbf{7 , 5 7 3 , 8 8 9}
\end{gathered}
$$

No. de encuestas

123

133

137

133

129

133

117

104

96

75

205

1,385

\section{Instrumento de Medición}

El instrumento utilizado es un cuestionario estructurado en un sólo bloque, que corresponde a la escala diseñada por Valence, d'Astous \& Fortier (Valence, 1988), y está compuesto de cuatro ítems para el constructo "Tendencia a gastar", cuatro ítems para el constructo "Urgencia de compra" y tres ítems para el constructo "Sentimientos de culpabilidad". El cuestionario se diseñó con una escala 1 a 5 tipo Likert, en donde 5 significa "totalmente de acuerdo" y 1 "totalmente en desacuerdo", en la escala diseñada por Valence, d'Astous \& Fortier los compradores compulsivos tienden a obtener calificaciones de 36 o más, ver tabla 2.

\section{Resultados}

\section{a) Análisis general del perfil demográfico de la muestra}

Participantes en el estudio: $60.1 \%$ mujeres y $39.9 \%$ hombres; $54.5 \%$ son personas solteras, $41.3 \%$ son casadas y $4.2 \%$ reporta otro estado civil. En lo que refiere a la ocupación, el $38 \%$ está empleado, el $15.7 \%$ es profesionista independiente, el $14.2 \%$ se ocupa en el hogar, el $13.6 \%$ es desempleado y el $18.6 \%$ son estudiantes. En cuanto a la educación $6.1 \%$ cuenta con primaria, $18.1 \%$ con secundaria, $27.7 \%$ con preparatoria o carrera técnica, $20.9 \%$ con licenciatura incompleta, $24 \%$ con licenciatura completa y $2.9 \%$ con estudios de posgrado. Finalmente, de las diez y seis delegaciones políticas del Distrito Federal, dos destacan en su participación, la delegación Coyoacán con el 18.6\% y la delegación Iztapalapa con el $13.1 \%$, el resto de las delegaciones registra una participación promedio de $4.9 \%$.

\footnotetext{
${ }^{2}$ INEGI, Censo poblacional 2010, Distrito Federal,
} 


\begin{tabular}{|c|c|}
\hline Constructo & Ítem \\
\hline Tendencia & $\begin{array}{r}\text { Compro cosas que no tenía planeado comprar } \\
\text { Cuando tengo dinero, no puedo contenerme y gasto una parte o todo } \\
\text { Cuando compro siento que se me quita la depresión } \\
\text { Cuando compro siento que obtengo felicidad }\end{array}$ \\
\hline Urgencia & $\begin{array}{c}\text { Con frecuencia soy impulsivo en mi comportamiento como comprador } \\
\text { En cuanto ingreso en un centro comercial, siento una necesidad } \\
\text { irresistible de entrar a alguna tienda a comprar algo } \\
\text { Soy una de esas personas que frecuentemente responden a las ofertas } \\
\text { enviadas por correo }\end{array}$ \\
& $\begin{array}{c}\text { Si me queda dinero al final del periodo de pago, siento que tengo que } \\
\text { gastarlo }\end{array}$ \\
\hline Culpabilidad & $\begin{array}{c}\text { En muchas ocasiones he comprado un artículo que no necesitaba, } \\
\text { sabiendo que tenía muy poco dinero disponible } \\
\text { Creo que otras personas se sentirían horrorizadas si conocieran mis } \\
\text { hábitos de gastos }\end{array}$ \\
& He comprado cosas que estaban fuera de mis posibilidades económicas \\
\hline
\end{tabular}

Tabla 2. Constructos e Ítems

\section{b) Nivel de compulsividad de la muestra}

\section{b.1 Escala de Edwards}

El continuo de compra compulsiva desarrollado por Edwards (Edwards, 1992) permite clasificar a los consumidores según el nivel de compulsión en la compra y puede también ayudar a estimar mejor la proporción de compradores compulsivos o casi compulsivos de una muestra. El continuo está construido con cinco niveles de compra: normal, recreacional, frontera, compulsividad y adicción. El promedio obtenido en la escala se muestran en la tabla 3.

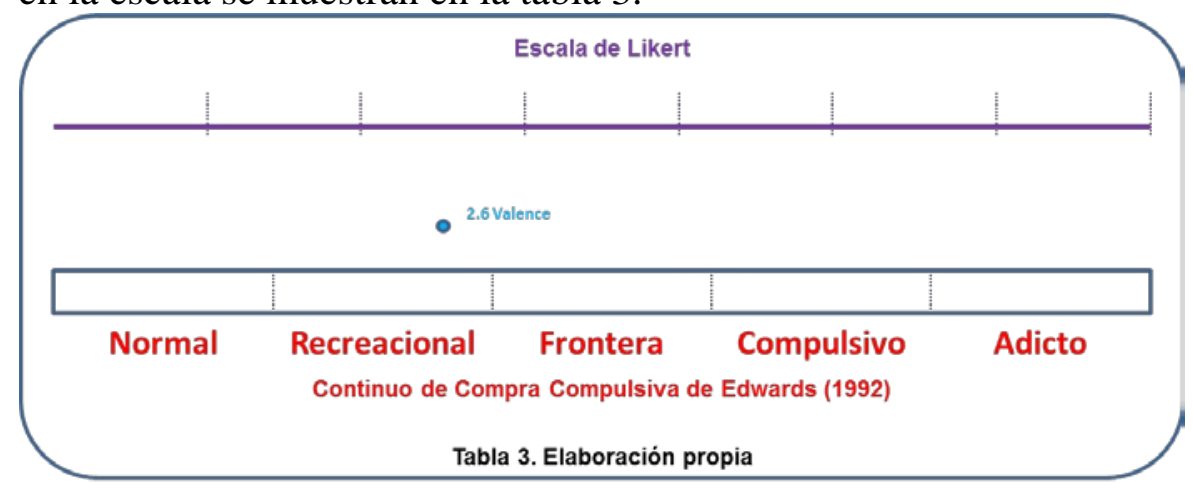

\section{b.2 Puntuación en la escala Valence, d'Astous \& Fortier}

El análisis de los constructos arroja las siguientes calificaciones promedio: Tendencia a gastar: 2.3, Urgencia de compra: 1.9, y Sentimientos de culpabilidad: 2.0. Considerando que la escala tiene un máximo de 5 en totalmente de acuerdo, los resultados obtenidos sugieren 
que los integrantes de la muestra no están centrados en la compra compulsiva; Este resultado inicial se confirma al aplicar la ecuación de puntuación de Valence, d'Astous \& Fortier (Valence, 1988), la cual arroja que solo el $18.8 \%$ de la muestra está representada por consumidores compulsivos, mientras que el $81.2 \%$ de la muestra $(1,166$ personas) la integran consumidores no-compulsivos. De este total (582) el $42 \%$ son hombres y el $58 \%$ son mujeres; el $54.5 \%$ son personas solteras y el 41.3 reportan ser casados; el 38\% son empleados, $15.6 \%$ son profesionistas y 14.2 se dedica al hogar; el $44.9 \%$ cuenta con estudios de licenciatura y el $51.8 \%$ con estudios intermedios.

\section{c) Correlación Producto-Momento de Pearson}

Se realizó un análisis de correlación Producto-Momento de Pearson con alfa de 0.05 en los constructos de la escala de Valence, d'Astous \& Fortier (Valence, 1988), los resultados encontrados se muestran en la tabla 4:

\begin{tabular}{|c|c|c|c|}
\hline \multicolumn{3}{|c|}{ Constructos escala Valence, d'Astous \& Fortier } \\
\hline & C1 & C2 & C3 \\
\hline C1 & & 0.72 & 0.63 \\
\hline C2 & & & 0.72 \\
\hline C3 & & \\
\hline C1 & Tendencia a gastar & \\
\hline C2 & Urgencia por comprar & \\
\hline C3 & Sentimiento de culpabilidad \\
\hline
\end{tabular}

Tabla 4. Elaboración propia

Se observa en la tabla 4 que hay correlación significativa del constructo "Tendencia a gastar" con los constructos "Urgencia por comprar" $\mathrm{r}=.72$, y "Sentimientos de culpabilidad” r=.63; así mismo, hay correlación significativa de los constructos "Urgencia por comprar" con "Sentimientos de culpabilidad” r=.72;

d) Comparación empírica de las medidas de compra compulsiva que proporcionan las escalas de Valence et al. (1988), Faber et al. (1992), y Ridgway (2008)

Los hallazgos logrados en esta investigación muestran similitud con los resultados obtenidos en nuestro primer estudio de consumo compulsivo realizado con habitantes de la ciudad de México (Fischer, L., Espejo, J., Méndez, A., Mellado, J 2015) y en el que se usó la escala de medición de Ridgway, Kular \& Monroe (Ridgway N. K., 2008), sin embargo, no existe similitud con los resultados de nuestro segundo estudio (Fischer et al., 2015) y en el que se usó la escala de medición de Faber, O'Guinn y Krych (Faber R. O., 1987, 1988a, 1988b, 1989, 1992). La tabla 5 muestra esta disimilitud mediante la comparación de los consumidores compulsivos y no compulsivos identificados a través de cada una de las escalas. 


\begin{tabular}{|c|c|c|}
\hline Escala & Compulsivos & No Compulsivos \\
\hline Ridgway, Kular \& Monroe & $152(11 \%)$ & $1,233(89 \%)$ \\
\hline Faber, O'Guinn y Krych & $1,071(77 \%)$ & $314(23 \%)$ \\
\hline Valence, d'Astous \& Fortier & $219(19 \%)$ & $1,166(81 \%)$ \\
\hline
\end{tabular}

Tabla 5. Elaboración propia

Estos hallazgos hacen evidente que las escalas se construyeron para diferentes propósitos, utilizando diferentes metodologías. La escala de Valence et al. (1988) parece más orientada a medir el grado de necesidad irresistible de comprar, mientras que la escala de Faber et al. (1992), está diseñada para capturar indicadores conductuales y financieros del comportamiento de compra compulsiva. Finalmente, la escala de Ridgway et al. (2008) se refiere específicamente a la acción de compra (Buying) y no a la experiencia de compra (Shopping), de acuerdo con Ridgway, después de todo, se puede ir de compras compulsivamente sin comprar; a diferencia de la escala de Faber, la escala de Ridgway se centra más en las características reales de la compra compulsiva que en las consecuencias.

\section{Conclusion y Consideraciones futuras}

En las últimas décadas la compra compulsiva ha dejado de ser un tema del humor sarcástico (por lo general a expensas del sexo femenino) para convertirse en un serio tema de estudio en las universidades, e incluso de gran preocupación estratégica para la mercadotecnia. Gracias a los estudios e investigaciones sobre el tema, en los últimos años los profesionales de la mercadotecnia han logrado una mejor comprensión de sus complejidades, sus diversas causas contributivas, la gravedad de sus efectos y el tamaño del problema. Sin embargo, todavía queda mucho por ser entendido, el presente estudio, junto con sus estudios hermanos, hacen única esta triada investigativa por su enfoque en el estudio comparativo de las características dimensionales y de rendimiento de las principales escalas de medición de la compra compulsiva, aplicadas a una muestra de los habitantes de la ciudad de México.

La compra compulsiva es una importante área de investigación en la mercadotecnia, su importancia deriva, en parte, de su naturaleza como un aspecto negativo de la conducta del consumidor. Sin embargo, una mayor investigación futura de los fenómenos negativos de consumo pueden proporcionar nuevas o modificadas perspectivas para estudiar y proponer conductas de consumo positivas. Por otra parte, la investigación sobre los aspectos negativos del consumo seguirá siendo útil, ya que potencialmente puede contribuir al bienestar de la sociedad, un criterio importante para la utilidad de cualquier investigación.

Para concluir, podemos decir que la compra compulsiva del consumidor mexicano es todavía un tema poco estudiado, pero 
afortunadamente creciente, por sus consecuencias disfuncionales, tanto psicológicas, sociales como financieras.

\section{References:}

AAP, A. P. (1985). Diagnostic and Statistical Manual of Mental Disorders. Washington, D.C.: American Psychiatric Association.

Arellano, R. (2002). Comportamiento del consumidor. México: Mc Graw Hill.

Babbie, E. (1988). Métodos de Investigación por encuesta. México: FCE Fondo de Cultura Económica.

Bandura, A. (1986). Social foundations of thought and action. New Jersey: Englewood Cliffs.

Blackwell, R. M. (2002). Comportamiento del consumidor $9^{a}$ Edición, 571 pp. México: Thomson.

Edwards, E. (1992). The measurement and modeling of compulsive consumer buying behavior. The University of Michigan. University Microfilms, Published Dissertation.

Faber, R. O. (1987, 1988a, 1988b, 1989, 1992). Compulsive consumption. Advances in Consumer Research, 14. Wallendorf and P. Anderson (Eds.), 132-135.

Faber, R. O. (1988). Compulsive Consumption. Advances in Consumer Research, Vol. 14, 132-35.

Faber, R. O. (1992). A Clinical Screener for Compulsive Buying. Journal of Consumer Research, 19 (December), 459-69.

Fischer, L. , Espejo, J(2011). Mercadotecnia. 4a Edición, 368 pp. Mexico: Mc Graw Hill.

Fischer, L., Espejo, J., Méndez, A., Mellado, J. (2015). El consumidor compulsivo en la ciudad de México, desde un enfoque de mercadotecnia de la salud. European Scientific Journal Oct, Ed. Vol 11 Num. 28, 101-11.

Fischer, L., Espejo, J., Méndez, A., Mellado, J. (2015). Existencia de compradores compulsivos en habitantes de la ciudad de méxico. European Scientific Journal, Vol 11, No 32 (2015): ESJ November Edition, 56-66.

García, I. (2007). Addictive Buying: Causes, Processes, and Symbolic Meanings. Thematic Analysis of a Buying Addict's Diary. The Spanish Journal of Psychology, 408-22.

Garnica, C. , Maubert, C.,. (2009). Fundamentos de Marketing. México: Pearson.

Goldenson, R. (1984). Longman Dictionary of Psychology and Psychiatry, 371 and 165;. New York: Longman.

Goodman, W. P. (1989). The Yale-Brown Obsessive Compulsive Scale. Psychiatry 46, 1006-11. 
Hawkins, I. B. (2004). Comportamiento del consumidor $9^{a}$ Edición, 758 pp. México: Mc Graw Hill.

Kotler, P. A. (2007). Marketing. Versión para Latinoamérica. Mexico: Pearson.

LaRose, R. E. (2002). Is online buying out of control? Electronic commerce and consumer self-regulation. . Journal of Broadcasting \& Electronic Media, 46(4), 549-64.

Luca-Arocas, R. (2000). Segmentos de consumidores según la escala de compra patológica. Investigación y Marketing Núm. 77, 16-25.

Monahan P, B. D. (1996). Reliability and validity of a scale to measure change in persons with compulsive buying. . Psychiatry Res. 64(1), 59-67.

O'Guinn, T. F. (1987). Purchasing not possessing: Aspects of materialism and compulsive consumption. American Psychological Association, 1-20.

Ridgway, N. K. (2008). An expanded conceptualization and a new measure of Compulsice Buying. Journal of Consumer Research, 622-39.

Roberts, J. J. (2001). Money attitudes, credit card use and compulsive buying among American college students. The Journal of Consumer Affairs, 35(21), 213-40.

Roberts, J. P. (2004). Personal goals and their role in consumer behavior: The case of compulsive buying. . Journal of Marketing Theory and Practice, Summer, 61-72.

Scheaffer, R. M. (2007). Elementos de Muestreo. España: Thomson.

Stanton, W. E. (2007). Fundamentos de Marketing 14 edición. México: McGraw Hill .

Valence, G. d. (1988). Compulsive Buying: Concept And Measurement. Journal of Consumer Policy, 11(4) , 419.

Workman, L. P. (2010). Compulsive buying: a theoretical framework. The Journal of Business Inquiry, 9, 1: 89-126. 